\title{
ON CONVEX FUNCTIONS OF HIGHER ORDER
}

\author{
AtTIla GILÁNYI AND Zsolt PÁLES
}

Abstract. Based on J. L. W. V. Jensen's concept of convex functions as well on its generalization by E. M. Wright and related to T. Popoviciu's convexity notions, higher-order convexity properties of real functions are introduced and surveyed.

Mathematics subject classification (2000): 26A51, 26A48, 39 B62.

Key words and phrases: Jensen-convexity, convexity of higher order, Wright-convexity.

\section{REFERENCES}

[1] E. F. Beckenbach, Generalized convex functions, Bull. Amer. Math. Soc. 43 (1937), 363-[371.

[2] S. Boyd, L. Vandenberghe, Convex Optimization, Cambridge University Press, Cambridge, 2004.

[3] P. S. Bullen, D. S. Mitrinović And P. M. Vasić, Means and Their Inequalities, D. Reidel Publ. Co., Dordrecht, 1988.

[4] Z. DARóCZY AND ZS. PÁLES, Convexity with given infinite weight sequences, Stochastica 11 (1987), no. 1, 5-12.

[5] B. De FinetTi, Sulle stratificazioni convesse, Ann. Mat. Pura Appl. [4] 30 (1949), 173-183.

[6] A. GILÁNYI AND Zs. PÁLES, On Dinghas-type derivatives and convex functions of higher order, Real Anal. Exchange 27 (2001/2002), 485-493.

[7] J. HADAMARD, Étude sur les propriétés des fonctions entières et en particulier d'une fonction considérée par Riemann, J. Math. Pures Appl. 58 (1893), 171-215.

[8] J. HADAMARD, Sur les fonctions entières, Bull. Soc. Math. France 24 (1896), 186-187.

[9] G. HAMEL, Eine Basis aller Zahlen und die unstetigen Lösungen der Funktionalgleichung $f(x+y)=$ $f(x)+f(y)$, Math. Ann. 60 (1905), 459-462.

[10] M. O. HöLDER, Über einen Mittelwertsatz, Nachr. Ges. Wiss. Göttingen, 1889, 38-47.

[11] J. L. W. V. JENSEN, Om konvekse funktioner og uligheder imellem middelvaerdier, Nyt. Tideskrift for Mathematik 16 B (1905), 49-69.

[12] J. L. W. V. JENSEN, Sur les fonctions convexes et les inégualités entre les valeurs moyennes, Acta Math. 30 (1906), 175-193.

[13] M. KuCzMA, An Introduction to the Theory of Functional Equations and Inequalities, Państwowe Wydawnictwo Naukowe - Uniwersytet Śląski, Warszawa-Kraków-Katowice, 1985.

[14] N. KuHN, A note on $t$-convex functions, General Inequalities, 4 (Oberwolfach, 1983) (W. Walter, ed.), International Series of Numerical Mathematics, vol. 71, Birkhäuser, Basel-Boston-Stuttgart, 1984, pp. 269-276.

[15] Gy. Maksa, K. Nikodem, And Zs. PÁles, Results on $t$-Wright convexity, C. R. Math. Rep. Acad. Sci. Canada 13 (1991), no. 6, 274-278.

[16] J. MATKOWSKI AND M. WRóBEL, A generalized a-Wright convexity and related functional equation, Ann. Math. Sil. (1996), no. 10, 7-12.

[17] D. S. Mitrinović, J. E. PEČArić, AND A. M. FinK, Classical and New Inequalities in Analysis, Kluwer Acad. Publ., Dordrecht, 1993.

[18] C. P. Niculescu And L.-E. Persson, Convex functions and their applications. A contemporary approach, CMS Books in Mathematics/Ouvrages de Mathmatiques de la SMC, 23. Springer, New York, 2006.

[19] K. NiKodem AND Zs. PÁLES, On $t$-convex functions, Real Anal. Exchange, 29 (2003/2004), 219-228. 
[20] T. Popoviciu, Sur quelques propriétés des fonctions d'une ou de deux variables réelles, Mathematica (Cluj) 8 (1934), 1-85.

[21] T. Popoviciu, Les fonctions convexes, Hermann et Cie, Paris, 1944.

[22] R. T. Rockafellar, Convex Analysis, Princeton University Press, Princeton, N. J. 1970.

[23] A. W. Roberts and D. E. VARberg, Convex Functions, Academic Press, New York-London, 1973.

[24] O. STOLZ, Grundzüge der Differential- und Integralrechnung I, Teubner, Leipzig, 1893.

[25] E. M. WRIGHT, An inequality for convex functions, Amer. Math. Monthly 61 (1954), 620-622. 